\title{
Osteopontin and osteoprotegerin in atherosclerotic plaque - are they significant markers of plaque vulnerability?
}

\author{
Cristina Strobescu-Ciobanu ${ }^{1,2)}$, Simona Eliza GiuşCă ${ }^{3)}$, IRINA-Draga CĂRUntu4),

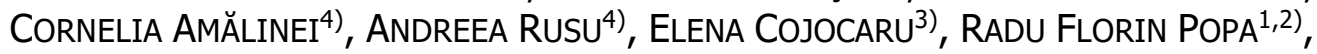 \\ CRISTIAN DUMITRU LUPAŞCU, ${ }^{5,6}$ \\ 1) Department of Vascular Surgery, Grigore T. Popa University of Medicine and Pharmacy, Iaşi, Romania \\ 2) Department of Vascular Surgery, Sf. Spiridon Emergency County Hospital, Iaşi, Romania \\ 3) Department of Morphofunctional Sciences I - Pathology, Grigore T. Popa University of Medicine and Pharmacy, \\ Iaşi, Romania \\ 4) Department of Morphofunctional Sciences I - Histology, Grigore T. Popa University of Medicine and Pharmacy, \\ Iaşi, Romania \\ 5) Department of Surgical Semiology, Grigore T. Popa University of Medicine and Pharmacy, Iaşi, Romania \\ 6) IId Surgery Department, Sf. Spiridon Emergency County Hospital, Iaşi, Romania
}

\begin{abstract}
Atherosclerosis (ATS) is still considered as a major, global health problem. For a deeper understanding of its pathogenesis, in the last years the research was translated from tissue visible events to molecular mechanisms. Osteopontin (OPN) and osteoprotegerin (OPG) are two molecules that have been associated with the initiation and progression of ATS lesions. The aim of our study was to assess the OPN and OPG expression in advanced stages of carotid ATS, to analyze the correlation between these markers and the ultrasonographic plaque properties, pointing out the identification of possible patterns that can predict plaque vulnerability and risks of restenosis. The study group comprised 49 consecutive patients ( 38 males and 11 females) diagnosed with carotid stenotic lesions by using ultrasonography. The carotid endarterectomy specimens were standardly processed for histopathological and immunohistochemical exams. The OPN and OPG expression was semi-quantitatively assessed. Our results sustained the relationship between histological American Heart Association (AHA) type and ultrasonographic classification (echogenic versus echolucent) $(p<0.001)$. The semi-quantitative analysis showed that in most cases ( 31 plaques) OPG and OPN had opposite expressions, whereas in the remaining cases (18 plaques) the expression was similar. There were no correlations between low versus high expression of intra-plaque OPN and OPG $(p=0.335)$. We found significant correlation for OPN and plaque echogenicity $(p=0.011)$, but not for OPG $(p=0.079)$. OPN expression (low versus high) was correlated with plaque type (stable versus unstable) $(p=0.036)$, plaque ulceration $(p=0.009)$ and inflammation $(p<0.001)$. OPG expression (low versus high) did not reveal statistically significant differences with plaque type (stable versus unstable) and vulnerability plaque parameters, respectively. OPG and OPN co-exist in carotid atherosclerotic plaque demonstrating a modulatory role in inflammatory and calcification processes. OPG is strongly expressed in stable, calcified plaques, while OPN is poorly expressed in calcified plaques and in plaques without hemorrhage, ulceration, inflammation, or necrosis. Starting from the molecular mechanisms, further studies of biomarkers are important to identify new therapeutic resources meant to prevent and treat vascular calcification.
\end{abstract}

Keywords: atherosclerosis, osteopontin, osteoprotegerin, ultrasonography, immunohistochemistry.

\section{口 Introduction}

More than 100 years after the first accepted definition [1], atherosclerosis (ATS) is still considered as a major, global health problem [2]. The outlining usually used for this chronic condition includes the following characteristics: it is a prominent cause of vascular disease, the main clinical manifestations are ischemic heart disease, stroke and peripheral arterial disease, and the key pathological feature is the thickening of the arterial wall [1]. The concept of atherosclerotic burden was introduced and agreed due to the impact of the disease on the general population, based on its predictive power for critical cardiovascular outcomes, followed by threatening complications and an increase rate of mortality $[2,3]$.

The deeper understanding of ATS pathology represents an important target in medical research. Remarkable results have been accumulated over time, leading to the identification and grouping of microscopic lesions in classifications whose relevance has been supported by clinicopathological correlations.

ATS has a progressive course affecting the large elastic and medium muscular arteries [4]. The pathognomonic lesion is the atherosclerotic plaque, which develops either by thickening resulting in stenosis or fissure of vessels, generating a thrombotic material involved in the occurrence of ischemic events [4]. Thus, the assessment of the atherosclerotic lesion type allows not only a staging but also an insight into the dynamics of the pathogenic process. Therefore, the most reliable classification of atherosclerotic lesions, developed by the American Heart Association (AHA), sets the type of lesion by microscopic examination

This is an open-access article distributed under the terms of a Creative Commons Attribution-NonCommercial-ShareAlike 4.0 International Public License, which permits unrestricted use, adaptation, distribution and reproduction in any medium, non-commercially, provided the new creations are licensed under identical terms as the original work and the original work is properly cited. 
of certain elements, closely related to the developmental stages of this process $[4,5]$.

There is a paradigm shift in understanding the ATS pathology in the last years, by translation from tissue visible events to molecular mechanisms [6-12]. Classical data were supplemented by the involvement of the endothelial, muscular, and inflammatory cell, through specific signaling pathways and molecular crosstalk [6-12].

Within this context, osteopontin (OPN) and osteoprotegerin (OPG) are two molecules that have been associated with the initiation and progression of ATS lesions, being linked to plaque vulnerability by modulating vascular calcification [13-15].

OPN is an extracellular matrix (ECM) protein that functions as a proinflammatory cytokine [16], beside binding potential of hydroxyapatite crystals to osteoblasts which has been originally described [17]. It is also involved in most systemic inflammatory processes and tissue remodeling [16]. The involvement of OPN in the pathogenic mechanism of ATS is supported by fibroblasts and macrophages ability to synthesize OPN $[16,18]$. Clinical evidence has confirmed a correlation between plasma OPN levels and coronary and aortic ATS, with a noticeable increased value in large vessel lesions [19].

OPG, a protein in the tumor necrosis factor receptor (TNFR) family, acts on bone tissue as an inhibitor of bone resorption and inhibits the immune response [20]. At cardiovascular level, OPG synthesized by macrophage and expressed by endothelial and smooth muscle cells has a protective role in the development of ATS [21]. OPG is one of the major components of endothelium-specific intracytoplasmic corpuscles, called Weibel-Palade bodies $[22,23]$. In endothelial activation by tumor necrosis factoralpha (TNF- $\alpha$ ) and interleukin- $1 \beta$ (IL-1 $\beta$ ), the corpuscles are secreted into the extracellular environment, thus protecting the endothelium from the apoptotic process triggered by the TNF-related apoptosis-inducing ligand (TRAIL) pathway [22, 23]. Moreover, OPG binds thrombospondin-1 (TSP-1), thus intervening in the regulation of vascular damage and thrombus formation [20]. OPG expressed by smooth muscle cells of medial layer is preventing the calcification of the ECM, but not the resorption of the already deposited calcium $[21,24]$.

Concurrently with the pathogenic mechanism of ATS, the researchers' interest is also directed on its clinical and therapeutic management. A critical problem is the fact that the disease can remain asymptomatic for many years, complications often being the first clinical manifestation.

The progress made in the development of non-invasive cardiovascular techniques, folded up by the increase of patients' accessibility to these techniques has improved the prognosis for ATS, allowing a more accurate evaluation of early stages of the disease. Ultrasonography (US) represents the first line approach for diagnosis. US performances allow not only the identification of the lesion, but also determine the degree of stenosis $[25,26]$ and the associated risk. It is already known that the echolucent plaques are vulnerable and with a higher probability to determine transitional ischemic strokes. Thus, imaging diagnosis can prevent dramatic ischemic lesions, by prioritizing those cases that require endarterectomy. Early diagnosis and therapy of atherosclerotic carotid artery disease have significantly reduced the risk of complications, mainly the stroke risk - the results being confirmed by two clinical trials targeting this issue $[27,28]$.

However, there are still limits related to the accuracy of imaging diagnosis. That is why researchers are testing additional markers that can enhance the quality of the diagnosis. Gray scale median (GSM) is an US marker for atherosclerotic plaque aspect $[29,30]$, but its value is still disputed in the literature.

Within this context of knowledge, the present research is based on the experience of the Department of Vascular Surgery, Sf. Spiridon Emergency County Hospital, Iaşi, Romania, in the diagnosis and surgical treatment of ATS lesions and completes our previous published reports on this subject $[31,32]$.

\section{Aim}

Specifically, our study aims to assess the OPN and OPG expression in advanced stages of carotid ATS, to analyze the correlation between these markers and the ultrasonographic plaque properties, pointing out the identification of possible patterns that can predict plaque vulnerability and risks of restenosis, after carotid endarterectomy (CEA).

\section{a Patients, Materials and Methods}

\section{Patients}

The study group comprised 49 consecutive patients ( 38 males and 11 females, average age $66 \pm 6.16$ years old) diagnosed with carotid stenotic lesions and treated in the Department of Vascular Surgery, Sf. Spiridon Emergency County Hospital, Iaşi, from January 2015 to April 2018. The selection of the patients was based on well-established inclusion criteria (namely 70-99\% stenosis of internal carotid arteries without symptoms, 50-99\% stenosis of internal carotid arteries associated with recent transitory ischemic attack or stroke). The exclusion criteria included the presence of chronic autoimmune, endocrine, hepatic, renal or cardiac conditions, and neoplasms. The study received the ethical approval of Grigore T. Popa University of Medicine and Pharmacy, Iaşi. Epidemiological, clinical characteristics, and preoperatory treatment for the patients in our group are summarized in Table 1.

\section{US imagistic evaluation}

The diagnosis was established using US, B-mode and Doppler [Siemens US equipment (X700)]. The examination of the common and internal carotid arteries was performed in supine position, the neck being rotated at $45^{\circ}$ in the opposite direction. The degree of stenosis was measured and reported according to North American Symptomatic Carotid Endarterectomy Trial (NASCET) method [25, 27, 31, 32].

The plaques were described and classified according to the Gray-Weale classes [31-33], in two main categories: vulnerable and stable. Vulnerable plaques included anechogenic plaques with fibrous cap (type 1) or plaques with less than $25 \%$ of echogenic areas (type 2 ), whereas stable plaques category included the hyperechogenic ones, with reduced anechogenic zones (less than 25\% - type 3) or with homogenous hyperechogenicity (type 4). After that, type 1 and type 2 plaques were grouped in echolucent plaques, whereas type 3 and $4-$ in echogenic plaques. 


\begin{tabular}{|c|c|c|}
\hline Characteristics & No. of cases & Percentage \\
\hline \multicolumn{3}{|l|}{ Gender } \\
\hline - Male & 38 & $77.5 \%$ \\
\hline - Female & 11 & $22.5 \%$ \\
\hline \multicolumn{3}{|l|}{ History of neurological symptoms } \\
\hline - TIA & 19 & $38.7 \%$ \\
\hline - Stroke & 18 & $36.7 \%$ \\
\hline - Amaurosis fugax & 3 & $6.1 \%$ \\
\hline - Absent & 9 & $18.4 \%$ \\
\hline \multicolumn{3}{|l|}{ Risk factors } \\
\hline - Hypertension & 37 & $75.5 \%$ \\
\hline - Dyslipidemia & 20 & $40.8 \%$ \\
\hline - Diabetes mellitus & 15 & $30.6 \%$ \\
\hline - Active smokers & 10 & $20.4 \%$ \\
\hline - Peripheral arterial disease & 15 & $30.6 \%$ \\
\hline \multicolumn{3}{|l|}{ Treatment } \\
\hline $\begin{array}{c}\text { - ASA/platelet aggregation } \\
\text { inhibitors before admission }\end{array}$ & 40 & $81.6 \%$ \\
\hline $\begin{array}{l}\text { - HMG-CoA reductase } \\
\text { inhibitors before admission }\end{array}$ & 48 & $97.9 \%$ \\
\hline
\end{tabular}

tetrahydrochloride chromogen (Abcam IHC detection kit, code: ab64261).

The expression of the two biomarkers was quantified taking into consideration the intensity of the immunolabeling (low and high, respectively) and the extension of the immunopositive area inside the plaque. Therefore, negative lesions and those with low immunopositivity in less than $50 \%$ of the plaque area were classified as low OPN/OPG expression, and the lesions with high OPN/OPG expression were considered when the immunostaining was noted in more than $50 \%$ of the interest area defined in the plaques. The assessment was performed by three trained histopathologists. The final results were decided in consensus, in order to avoid the degree of subjectivity in the semi-quantitative assessment.

\section{Statistical analysis}

For statistical analysis, we used the Statistical Package for the Social Sciences (SPSS) program (version 19.0). Nominal variables were analyzed using the $\chi^{2}$ (chi-squared) test and we considered a priori a $p$-value $<0.05$ as significant.

\section{ㅁ Results}

\section{Imaging characteristics}

The results of ultrasonographic examination revealed 33 patients who presented a degree of stenosis between $70-99 \%, 14$ patients with stenosis between $60-69 \%$, and two patients with stenosis between $50-59 \%$.

All identified internal or common carotid lesions were classified as follows: 21 plaques as echolucent, and 28 plaques as echogenic. We noticed that most of the echolucent plaques were type 1 (16 cases), anechogenic with echogenic fibrous cap, whereas the echogenic plaques were predominantly type 3 (26 cases), characterized by hyperechogenicity mixed with less than $25 \%$ anechogenic zones.

By using the GSM score and a threshold of 37 based on the GSM value for quartile 25, 13 plaques were considered vulnerable (GSM <37), and 36 plaques were stable (GSM $>37)$.

\section{Histopathological characteristics}

The microscopic exam showed 35 plaques with specific features for $A H A$ type V (morphologically stable plaques) and 14 plaques with particular morphological changes for $A H A$ type VI (morphologically unstable plaques).

\section{OPN and OPG expression}

The qualitative assessment of OPN and OPG in atherosclerotic plaques showed a fine granular expression pattern, with heterogeneous distribution and variable intensity (low, moderate, or high).

OPN expression, with low or moderate intensity, was identified in the lipid core and in the areas adjacent to dystrophic calcifications in more than $50 \%$ of cases (Figure 1). High intensity expression of OPN was observed in the lipid core and very rarely in the ECM, only in plaques with moderate or well represented inflammatory infiltrate (Figure 2). Cells expressing OPN were macrophages, myofibroblasts, and focal smooth muscle cells, but the intensity of expression was low. 
OPG high intensity expression was constantly identified in the intimal layers as fine granular areas within dystrophic calcification and around them, as well as in atheromatous core (Figures 3 and 4). OPG was also identified in the ECM of plaques, occupying large areas (corresponding to increased synthesis), especially in plaques with associated inflammation or numerous smooth muscle cells. Areas containing foamy macrophages and smooth muscle cells in the media were strongly positive for OPG. OPG was

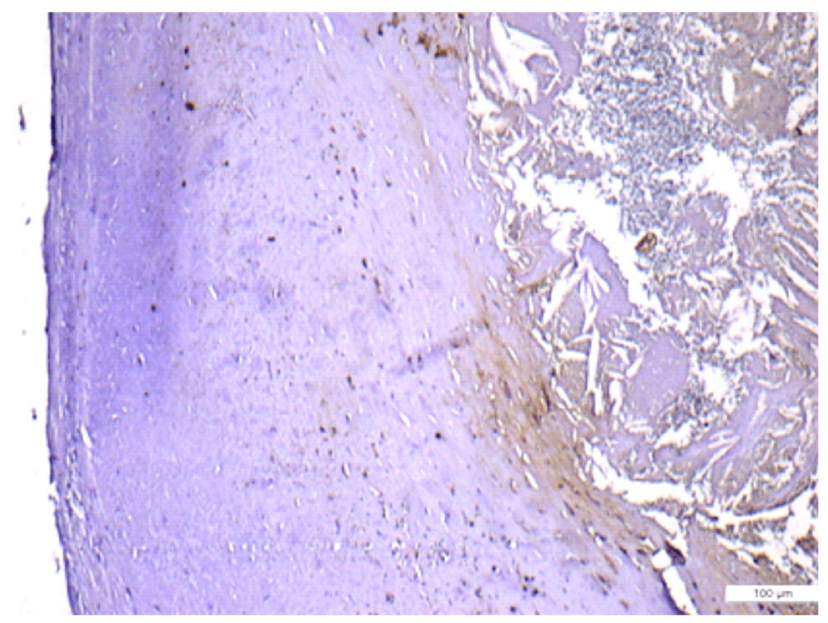

Figure 1 - Low OPN expression in the periphery of the lipid core (IHC staining with anti-OPN antibody, $\times 100$ ). IHC: Immunohistochemical; OPN: Osteopontin.

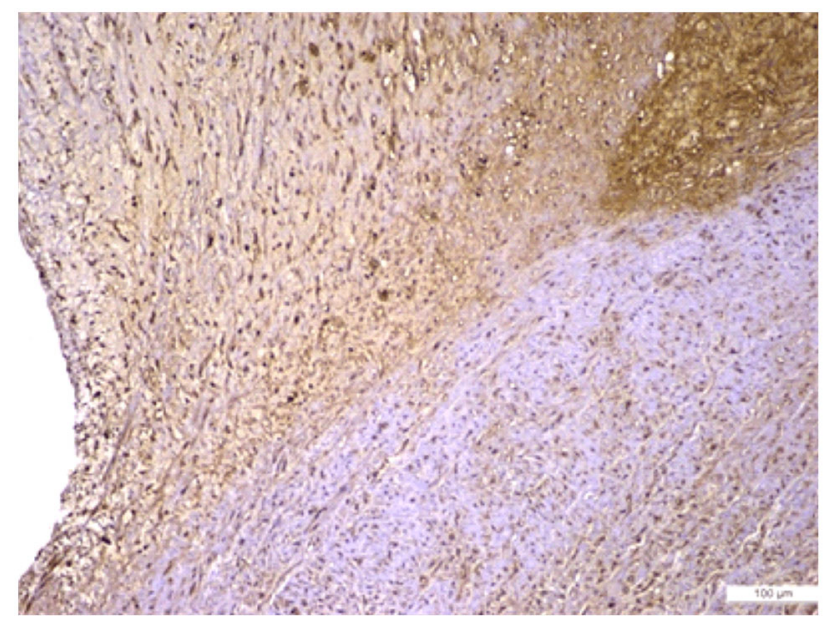

Figure 3 -Strong OPG expression in the fibro-lipid plaque (lipid core, smooth muscle cells, fibroblasts, extracellular matrix); smooth muscle cells OPG positive in the media (IHC staining with anti-OPG antibody, $\times 100)$. IHC: Immunohistochemical; OPN: Osteoprotegerin.

\section{Clinico-pathological and imaging correlations}

A significant statistical correlation was noticed between histological $A H A$ type and ultrasonographic classification (echogenic versus echolucent) $(p<0.001)$.

Ultrasonographic classification was correlated with hemorrhage $(p=0.013)$, ulceration $(p=0.001)$, inflammation $(p=0.004)$, and necrosis $(p=0.025)$, but no significant correlation was registered for the plaque calcification $(p=0.219)$. also focally observed in smooth muscle cells migrated to the fibrous cap. OPG decreased cellular expression was associated with increased expression of OPG in the ECM.

The semi-quantitative assessment based on the applied scoring system, revealed low OPN expression in 35 plaques and high expression in the other 14 plaques specimens. On the other hand, OPG was low in 16 plaques and high in 33 plaque specimens.

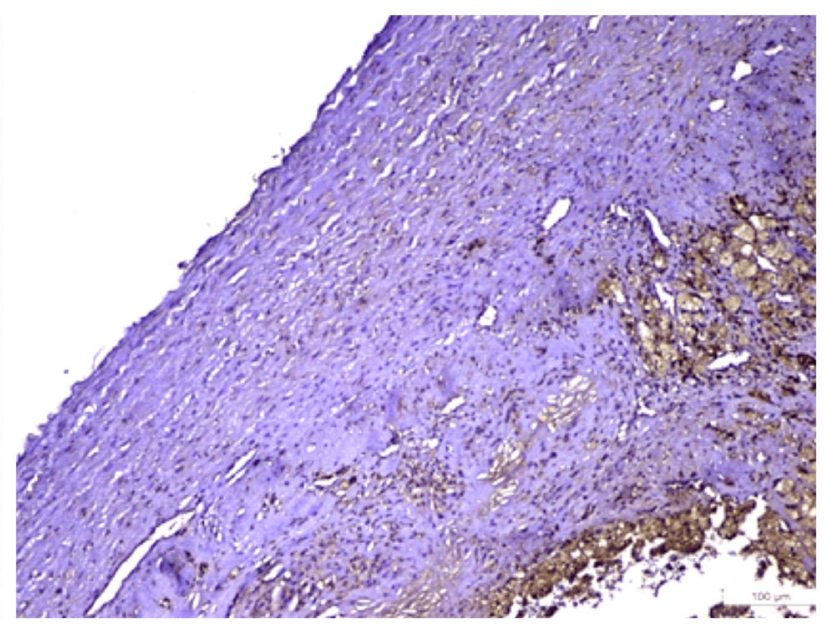

Figure 2 - Strong OPN expression in the periphery of the calcification area, in foamy macrophages, fibroblasts, and inflammatory cells of the plaque (IHC staining with anti-OPN antibody, $\times 100)$. IHC: Immunohistochemical; OPN: Osteopontin.

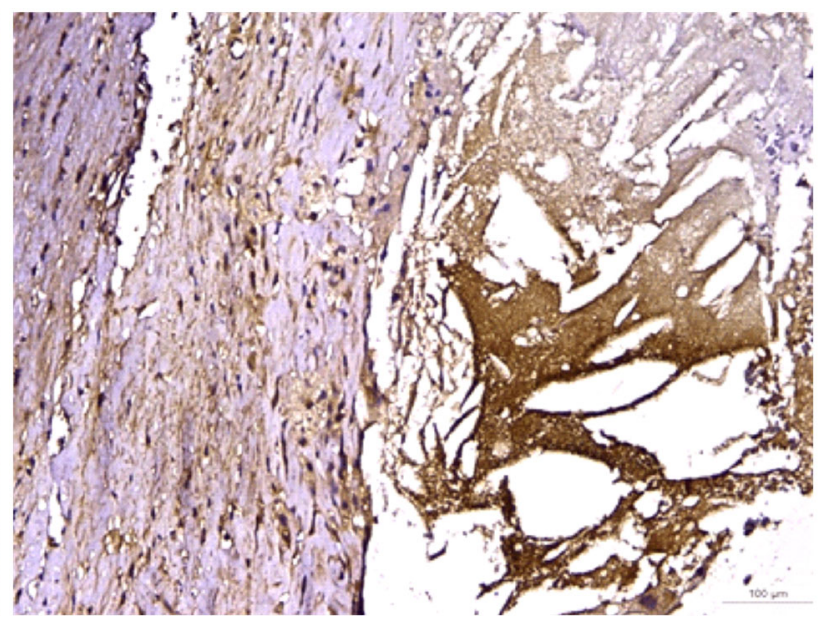

Figure 4 - Strong OPG expression in the lipid core, adjacent to cholesterol crystals, in the extracellular matrix and intracellular matrix resident cells (fibroblasts) or migrated cells (inflammatory elements) (IHC staining with anti-OPG antibody, $\times 100)$. IHC: Immunohistochemical; OPN: Osteoprotegerin.

There were no statistically significant differences between low versus high expression of intra-plaque OPN and OPG $(p=0.335)$.

Statistical analysis showed significant correlation between OPN and plaque echogenicity ( $p=0.011$ ), but not for OPG $(p=0.079)$. Moreover, no statistical significance could be identified between OPG or OPN and GSM, neither as individual value nor as threshold.

Statistically significant differences were registered 
between OPN expression (low versus high) and plaque type (stable versus unstable) $(p=0.036)$ (Table 2$)$, and also for plaque ulceration $(p=0.009)$ and inflammation $(p<0.001)$.
OPG expression (low versus high) did not reveal statistically significant differences with plaque type (stable versus unstable) and vulnerability plaque parameters, respectively (Table 2 ).

Table 2 - OPN and OPG expression in correlation to morphological parameters

\begin{tabular}{|c|c|c|c|c|c|c|c|}
\hline \multirow{2}{*}{\multicolumn{2}{|c|}{ Morphological parameters }} & \multicolumn{2}{|c|}{ OPN expression } & \multirow{2}{*}{$\stackrel{p}{x^{2}} \stackrel{\text { test }}{ }$} & \multicolumn{2}{|c|}{ OPG expression } & \multirow{2}{*}{$\stackrel{p}{X^{2} \text { test }}$} \\
\hline & & Low & High & & Low & High & \\
\hline \multirow{2}{*}{ Calcification } & Negative & $6(60 \%)$ & $4(40 \%)$ & \multirow{2}{*}{0.370} & $2(20 \%)$ & $8(80 \%)$ & \multirow{2}{*}{0.339} \\
\hline & Positive & $29(74.4 \%)$ & $10(25.6 \%)$ & & $14(35.9 \%)$ & $25(64.1 \%)$ & \\
\hline \multirow{2}{*}{ Hemorrhage } & Negative & $31(73.8 \%)$ & $11(26.2 \%)$ & \multirow{2}{*}{0.366} & $15(35.7 \%)$ & $27(64.3 \%)$ & \multirow{2}{*}{0.263} \\
\hline & Positive & $4(57.1 \%)$ & $3(42.9 \%)$ & & $1(14.3 \%)$ & $6(85.7 \%)$ & \\
\hline \multirow{2}{*}{ Ulceration } & Negative & $30(81.1 \%)$ & $7(18.9 \%)$ & \multirow{2}{*}{0.009} & $14(37.8 \%)$ & $23(62.2 \%)$ & \multirow{2}{*}{0.174} \\
\hline & Positive & $5(41.7 \%)$ & $7(58.3 \%)$ & & $2(16.7 \%)$ & $10(83.3 \%)$ & \\
\hline \multirow{2}{*}{ Inflammation } & Negative & $29(90.6 \%)$ & $3(9.4 \%)$ & \multirow{2}{*}{$<0.001$} & $11(34.4 \%)$ & $21(65.6 \%)$ & \multirow{2}{*}{0.724} \\
\hline & Positive & $6(35.3 \%)$ & $11(64.7 \%)$ & & $5(29.4 \%)$ & $12(70.6 \%)$ & \\
\hline \multirow{2}{*}{ Necrosis } & Negative & $27(75 \%)$ & $9(25 \%)$ & \multirow{2}{*}{0.357} & $13(36.1 \%)$ & $23(63.9 \%)$ & \multirow{2}{*}{0.390} \\
\hline & Positive & $8(61.5 \%)$ & $5(38.5 \%)$ & & $3(23.1 \%)$ & $10(76.9 \%)$ & \\
\hline \multicolumn{2}{|c|}{ Morphologically stable plaques } & $28(80 \%)$ & $7(20 \%)$ & \multirow{2}{*}{0.036} & $13(37.1 \%)$ & $22(62.9 \%)$ & \multirow{2}{*}{0.289} \\
\hline \multicolumn{2}{|c|}{ Morphologically unstable plaques } & $7(50 \%)$ & $7(50 \%)$ & & $3(21.4 \%)$ & $11(78.6 \%)$ & \\
\hline
\end{tabular}

OPG: Osteoprotegerin; OPN: Osteopontin.

\section{ㅁ Discussions}

The value of the histological analysis of carotid endarterectomy specimens versus the imaging techniques

Currently, plaques removed by CEA which are not analyzed by standard histology are wasted. Several studies have emphasized that the histological analysis of the plaque specimens harvested during a CEA may provide insights in the atherosclerotic process of the entire vascular system $[34,35]$. It is worth mentioning that the accurate diagnosis for ATS lesions is based on the histological exam, in accordance with $A H A$ classification. Thus, the unstable plaques, susceptible to rupture have a central necrosis mass, rich inflammatory infiltrate, and a thin fibrous cap. On the other hand, the stable plaques show a small lipid core, few inflammatory cells, and a thick fibrous cap. The histological characterization of the CEA specimens can be compared with imaging features of the plaques, leading to a more precise diagnosis for stable versus vulnerable plaques. These features have major implications in the prediction of the atherosclerotic process evolution, including the possible risk of a cerebral thromboembolism resulting in stroke, transient ischemic attacks or amaurosis fugax $[36,37]$. We must highlight that in our study most carotid atherosclerotic plaques have been obtained from asymptomatic patients (either with no symptomatology at all or with cerebral ischemic events older than six months) and were echogenic, stabile plaques, with surgical indications for CEA due to stenosis severity.

The review of the literature reveals constant effort aiming to provide uniformity in reports on atherosclerotic plaque imaging [either US, computed tomography (CT) or magnetic resonance imaging (MRI)] versus histology.

One of the first relevant paper on this topic, published in the 80 's, reported that the echolucent plaques frequently present intraplaque hemorrhage in comparison with the echogenic ones [33].
Another classification is based on the degree of plaque echogenicity, proposing three distinct types: hypoechogenic plaque, often associated with serious retinal phenomena, hyperechogenic plaque, correlated with an asymptomatic status, and intermediate echoic plaque, responsible for the occurrence of cerebrovascular symptoms [38]. The presence of hemorrhage area represents a characteristic of hypoechogenic plaques, whereas necrosis and calcification do not have an echomorphologic predilection [38]. This makes it difficult to distinguish by ultrasound techniques between calcified plaques and those with necrosis, thus limiting the diagnostic accuracy of this investigation and suggesting the need for additional histological analysis [38].

The comparative histological-imaging approach could provide a new perspective in establishing the type of atherosclerotic lesions by promoting a non-invasive investigation.

Relevant data are provided by a review that analyzed 73 studies that compared carotid plaque imaging with carotid plaque histology [34]. Unfortunately, this review revealed a large variety of histological and/or imaging techniques, including performance of the equipment and the examiner subjectivity, without a common methodology and measurement parameters. Therefore, the paper cannot offer an integrated, histological-imaging overview regarding the atheromatous process [34]. Histological assessment had been variable according to the processing method, type of section (cross-section in $66 \%$ and longitudinally in $4 \%$ of studies, respectively), thickness of the sample, staining, and not least the number of sections [34]. From the total number of studies, the correlations between histology and imaging addressed the size of lipid core by reference to the fibrous layer in $77 \%$, calcification in $66 \%$, intra-plaque hemorrhage in $56 \%$, surface-thrombus in $37 \%$, rupture in $21 \%$, inflammation in $12 \%$, cap thickness in $10 \%$, and plaque neovascularization in $4 \%$ [34]. The evaluation was predominantly qualitative and only 20 studies used 
computer-assisted image analysis in addition to classical histological exam to determine the percentage of each element in the structure of plaque [34]. On the other hand, even if the same imaging methods were used, with similar criteria ensuring the reproducibility of the study (i.e., Gray-Weale scale of ultrasonographic echogenicity), the results were extremely variable [34].

All these deficiencies led to the necessity to develop new reporting criteria that show atheromatous plaque characteristics from both histological and imaging point of view, in order to provide a diagnosis as accurate as possible [34]. The correlation between the histological analysis and the imaging technique (US, CT or MRI) facilitates the atheromatous process staging and enables the development of appropriate therapeutic strategy in relation to the particularities of each reported investigation.

Relying on the classification of atherosclerotic plaques in echogenic or echolucent, our study identifies a strong correlation with histological examination and $A H A$ plaque type, providing information about carotid plaque vulnerability by identifying hemorrhage $(p=0.013)$, ulceration $(p=0.001)$, inflammation $(p=0.004)$ or necrosis $(p=0.025)$ in echolucent plaques.

Although other studies showed significant correlation between high echogenicity and calcified plaques [39, 40], our study did not confirm this association. Our results indicate that the echogenic plaques are predominantly calcified and also some of the echolucent, vulnerable plaques show a variable degree of calcification.

Thus, imaging diagnosis does not equal the specific ability of histopathology to refine lesions type and there is no perfect matching between US and the histological approach. US offer a two-dimensional image, with low potential to cover the entire plaque volume and it is dependent on the potential to overcome shadowing artefacts caused by calcified plaques [41]. Unfortunately, US is characterized by low levels of reproducibility [42, 43] and GSM values have a very low level of correlation with HP examination results [44].

The optimization of the imaging techniques is an important step to promote non-invasive methods for diagnosis, but it cannot be done in the absence of histological contributions. Early diagnosis of atherosclerotic lesions and their predicable evolution are common goals of clinical and histological examinations. Any success in this approach provides new perspectives for a deeper understanding of ATS, a disease with significant incidence and impact in general population.

\section{The significance of OPN and OPG expression}

Classically, the etiopathogenesis of ATS involves metabolic, mechanical, infectious, or inflammatory lesions $[4,11]$. Nowadays, current trends in ATS research trigger the molecular pathogenesis that determines the onset and progression of disease. From the description of specific histological changes, the level of knowledge has deepened into specific mechanisms and pathways based on the synergistic action of products synthesized by vascular wall cells and circulating factors [45]. Despite the progress made in deciphering the molecular interferences that lead to vascular damage and structural remodeling, there are still a multitude of unknown elements.
The extensive panel of molecular markers involved in the pathogenic mechanism of ATS includes OPN and OPG, considered as calcification markers. These bonematrix proteins seem to have an important role in mineral deposition and osteoclastogenesis inhibition and are expressed by most vascular cells [13, 46, 47].

OPN is synthesized mainly by macrophages stimulated by numerous cytokines [i.e., angiotensin II, TNF- $\alpha$, interferon-gamma (IFN- $\gamma$ ), and transforming growth factor beta (TGF- $\beta$ ), and various interleukins - IL-1, IL-2, IL-3], lipopolysaccharides and nitric oxide [48]. Although OPN upregulation is still unknown, it seems that peroxisome proliferator-activated receptor gamma (PPAR $\gamma)$ has the main role, as an inhibitor of its gene expression. OPN acts by direct interaction with several types of membrane receptors belonging to integrin family and with cluster of differentiation 44R (CD44R) [49]. Once synthesized, OPN is responsible for several actions: it promotes adhesion, migration, and monocyte differentiation, stimulates matrix metalloproteinase (MMP)-2 and MMP-9 expression, stimulates phenotypic T1-lymphocytes differentiation, and inhibits T2-lymphocytes differentiation. Given that MMPs are involved in degradation of the ECM, this effect is synergistic with the pro-migratory monocyte action [50]. Thus, OPN has a crucial role in the recruitment of macrophages which, in turn, stimulate cytokine secretion and, consequently, cellular immunity is activated [16]. As a chemoattractant for inflammatory cells, OPN has an important role in healing and in vascular remodeling, and, supplementary, it has an inhibitory effect on calcification [51]. Consequently, OPN could be responsible for the evolution of unstable plaques $[46,47,52,53]$.

A close relationship between plasma OPN levels and ATS has been demonstrated [19]. In vulnerable plaques, increased OPN expression is associated with the association of inflammation and calcifications [18, 54].

The major role of OPG is associated with bone turnover [20]. The study of OPG expression in normal and pathological status is also demonstrating other functions, such as: promoter of cell survival, stimulator of tumor cells apoptosis and inhibitor of the immune response. The process of vascular calcification during ATS lesions progression has many similitudes with bone formation, without being identical phenomena [20, 24].

The role of OPG in the induction of osteogenesis analyzed by several experimental and clinical studies reveal contradictory data. Some reports indicate that the presence of OPG inhibits the mineralization process, thus having a potential role in preventing calcification in atheroma plaques $[22,24,55]$. According to these data, the fibrous cap plaques are dominant in carotids $(73 \%)$ and express more OPG, while calcified plaques are more common in femorals (93\%) and show a lower OPG expression [55]. Increased OPG expression in carotid plaques is correlated with an increased number of macrophages [22]. On the other hand, other reports show an increase in morbidity and mortality by cardiovascular disease associated with elevated serum OPG levels, as well as an increased OPG expression in unstable plaques [56-58]. Thus, although OPG is unanimously accepted as a marker of ATS and of intraplaque stabilization, it is not clearly established whether it is a pathogenic factor or appears because of endothelial activation [56, 58, 59]. 
Our semi-quantitative analysis of OPN and OPG in atherosclerotic plaque, defining the expression as low and high, showed that in most cases (31 plaques) OPG and OPN had opposite expressions. However, OPG and OPN expression was similar in 18 cases (eight cases high and 10 cases low expression, respectively). These data support that OPG and OPN are co-expressed in carotid atherosclerotic plaques, as markers of inflammation and calcification processes.

From a clinical and histopathological point of view, the presence of calcification in the carotid plaque determines its stability and decreases the risk of rupture and the presence of symptomatology.

Our data support the dual role of the OPN. OPN was poorly expressed in 29 calcified plaques and in plaques without hemorrhage ( 31 plaques), ulceration (30 plaques), inflammation (29 plaques) or necrosis (27 plaques). The correlation of OPN expression with the presence of intraplaque ulceration and inflammation confirms its involvement in vulnerable morphological lesions ( $p=0.036$ ).

Our data support the association of OPG with the mineralization process, OPG being strongly expressed in stable, calcified plaques. More specifically, OPG was highly positive in 33 plaques, 25 (75\%) of them showing calcifications in histological examination.

In addition to these data, our study shows no statistical significance between GSM and $A H A$ plaques type or the presence of OPN or OPG.

\section{Conclusions}

OPG and OPN co-exist in carotid atherosclerotic plaque demonstrating a modulatory role in inflammatory and calcification processes. OPG is strongly expressed in stable, calcified plaques, while OPN is poorly expressed in calcified plaques and in plaques without hemorrhage, ulceration, inflammation, or necrosis.

\section{Conflict of interests}

The authors declare that they have no conflict of interests.

\section{References}

[1] Adebayo O, Adeoye AM. Atherosclerosis: a journey around the terminology. In: Gianturco L (ed). Atherosclerosis, arteriosclerosis and arteriolosclerosis. IntechOpen, 2020. https://doi.org/10. 5772/intechopen.91064

[2] Herrington W, Lacey B, Sherliker P, Armitage J, Lewington S Epidemiology of atherosclerosis and the potential to reduce the global burden of atherothrombotic disease. Circ Res, 2016, 118(4):535-546. https://doi.org/10.1161/CIRCRESAHA. 115.307611 PMID: 26892956

[3] Forouzanfar MH, Moran AE, Flaxman AD, Roth G, Mensah GA, Ezzati M, Naghavi M, Murray CJ. Assessing the global burden of ischemic heart disease, part 2: analytic methods and estimates of the global epidemiology of ischemic heart disease in 2010. Glob Heart, 2012, 7(4):331-342. https://doi. org/10.1016/j.gheart.2012.10.003 PMID: 23505617 PMCID: PMC3595103

[4] Otsuka F, Yasuda S, Noguchi T, Ishibashi-Ueda H. Pathology of coronary atherosclerosis and thrombosis. Cardiovasc Diagn Ther, 2016, 6(4):396-408. https://doi.org/10.21037/cdt.2016. 06.01 PMID: 27500096 PMCID: PMC4960071

[5] Stary HC, Chandler AB, Dinsmore RE, Fuster V, Glagov S, Insull W Jr, Rosenfeld ME, Schwartz CJ, Wagner WD, Wissler RW. A definition of advanced types of atherosclerotic lesions and a histological classification of atherosclerosis. A report from the Committee on Vascular Lesions of the
Council on Arteriosclerosis, American Heart Association. Circulation, 1995, 92(5):1355-1374. https://doi.org/10.1161/ 01.cir.92.5.1355 PMID: 7648691

[6] Rader DJ, Daugherty A. Translating molecular discoveries into new therapies for atherosclerosis. Nature, 2008, 451(7181): 904-913. https://doi.org/10.1038/nature06796 PMID: 18288179

[7] Libby P, Ridker PM, Hansson GK. Progress and challenges in translating the biology of atherosclerosis. Nature, 2011, 473(7347):317-325. https://doi.org/10.1038/nature10146 PMID: 21593864

[8] Pedicino D, Giglio AF, Galiffa VA, Cialdella P, Trotta F, Graziani F, Liuzzo G. Infections, immunity and atherosclerosis: pathogenic mechanisms and unsolved questions. Int J Cardiol, 2013, 166(3):572-583. https://doi.org/10.1016/j.ijcard.2012. 05.098 PMID: 22727974

[9] Tabas I, García-Cardeña G, Owens GK. Recent insights into the cellular biology of atherosclerosis. J Cell Biol, 2015, 209(1):13-22. https://doi.org/10.1083/jcb.201412052 PMID: 25869663 PMCID: PMC4395483

[10] Brown RA, Shantsila E, Varma C, Lip GYH. Current understanding of atherogenesis. Am J Med, 2017, 130(3):268282. https://doi.org/10.1016/j.amjmed.2016.10.022 PMID: 27888053

[11] Wang T, Butany J. Pathogenesis of atherosclerosis. Diagn Histopathol, 2017, 23(11):473-478. https://doi.org/10.1016/j. mpdhp.2017.11.009

[12] Nakahara T, Dweck MR, Narula N, Pisapia D, Narula J, Strauss HW. Coronary artery calcification: from mechanism to molecular imaging. JACC Cardiovasc Imaging, 2017, 10(5): 582-593. https://doi.org/10.1016/j.jcmg.2017.03.005 PMID: 28473100

[13] Abedin M, Tintut Y, Demer LL. Vascular calcification: mechanisms and clinical ramifications. Arterioscler Thromb Vasc Biol, 2004, 24(7):1161-1170. https://doi.org/10.1161/ 01.ATV.0000133194.94939.42 PMID: 15155384

[14] Collin-Osdoby P. Regulation of vascular calcification by osteoclast regulatory factors RANKL and osteoprotegerin. Circ Res, 2004, 95(11):1046-1057. https://doi.org/10.1161/ 01.RES.0000149165.99974.12 PMID: 15564564

[15] Kadoglou NPE, Gerasimidis T, Moumtzouoglou A, Kapelouzou A Sailer N, Fotiadis G, Vitta I, Katinios A, Kougias P, Bandios S, Voliotis K, Karayannacos PE, Liapis CD. Intensive lipid-lowering therapy ameliorates novel calcification markers and GSM score in patients with carotid stenosis. Eur J Vasc Endovasc Surg, 2008, 35(6):661-668. https://doi.org/10.1016/j.ejvs.2007.12. 011 PMID: 18395477

[16] Kahles F, Findeisen HM, Bruemmer D. Osteopontin: a novel regulator at the cross roads of inflammation, obesity and diabetes. Mol Metab, 2014, 3(4):384-393. https://doi.org/ 10.1016/j.molmet.2014.03.004 PMID: 24944898 PMCID: PMC4060362

[17] Denhardt DT, Noda M. Osteopontin expression and function: role in bone remodeling. J Cell Biochem Suppl, 1998, 72(S30-31):92-102. PMID: 9893260

[18] Kwon HM, Hong BK, Kang TS, Kwon K, Kim HK, Jang Y, Choi D, Park HY, Kang SM, Cho SY, Kim HS. Expression of osteopontin in calcified coronary atherosclerotic plaques. J Korean Med Sci, 2000, 15(5):485-493. https://doi.org/10. 3346/jkms.2000.15.5.485 PMID: 11068982 PMCID: PMC 3054686

[19] Momiyama Y, Ohmori R, Fayad ZA, Kihara T, Tanaka N, Kato R, Taniguchi H, Nagata M, Nakamura H, Ohsuzu F. Associations between plasma osteopontin levels and the severities of coronary and aortic atherosclerosis. Atherosclerosis, 2010, 210(2):668-670. https://doi.org/10.1016/ j.atherosclerosis.2009.12.024 PMID: 20074733 PMCID: PMC3095031

[20] Reid P, Holen I. Pathophysiological roles of osteoprotegerin (OPG). Eur J Cell Biol, 2009, 88(1):1-17. https://doi.org/10. 1016/j.ejcb.2008.06.004 PMID: 18707795

[21] Scatena M, Liaw L, Giachelli CM. Osteopontin: a multifunctional molecule regulating chronic inflammation and vascular disease. Arterioscler Thromb Vasc Biol, 2007, 27(11):2302-2309. https://doi.org/10.1161/ATVBAHA.107.144824 PMID: 17717292

[22] Heymann MF, Herisson F, Davaine JM, Charrier C, Battaglia S, Passuti N, Lambert G, Gouëffic Y, Heymann D. Role of the OPG/RANK/RANKL triad in calcifications of the atheromatous plaques: comparison between carotid and femoral beds. 
Cytokine, 2012, 58(2):300-306. https://doi.org/10.1016/j.cyto. 2012.02.004 PMID: 22402034

[23] Rochette L, Meloux A, Rigal E, Zeller M, Cottin Y, Vergely C. The role of osteoprotegerin and its ligands in vascular function. Int J Mol Sci, 2019, 20(3):705. https://doi.org/10.3390/ijms 20030705 PMID: 30736365 PMCID: PMC6387017

[24] Van Campenhout A, Golledge J. Osteoprotegerin, vascular calcification and atherosclerosis. Atherosclerosis, 2009, 204(2): 321-329. https://doi.org/10.1016/j.atherosclerosis.2008.09.033 PMID: 19007931 PMCID: PMC2729052

[25] Oates CP, Naylor AR, Hartshorne T, Charles SM, Fail T, Humphries K, Aslam M, Khodabakhsh P. Joint recommendations for reporting carotid ultrasound investigations in the United Kingdom. Eur J Vasc Endovasc Surg, 2009, 37(3):251-261. https://doi.org/10.1016/j.ejvs.2008.10.015 PMID: 19046904

[26] Naylor AR, Ricco JB, de Borst GJ, Debus S, de Haro J, Halliday A, Hamilton G, Kakisis J, Kakkos S, Lepidi S, Markus HS, McCabe DJ, Roy J, Sillesen H, van den Berg JC, Vermassen F, ESVS Guidelines Committee, Kolh P, Chakfe N, Hinchliffe RJ, Koncar I, Lindholt JS, Vega de Ceniga M, Verzini F, ESVS Guideline Reviewers, Archie J, Bellmunt S, Chaudhuri A, Koelemay M, Lindahl AK, Padberg F, Venermo M. Editor's Choice - Management of atherosclerotic carotid and vertebral artery disease: 2017 Clinical Practice Guidelines of the European Society for Vascular Surgery (ESVS). Eur J Vasc Endovasc Surg, 2018, 55(1):3-81. https://doi.org/10.1016/j.ejvs.2017. 06.021 PMID: 28851594

[27] North American Symptomatic Carotid Endarterectomy Trial Collaborators; Barnett HJM, Taylor DW, Haynes RB, Sackett DL, Peerless SJ, Ferguson GG, Fox AJ, Rankin RN, Hachinski VC, Wiebers DO, Eliasziw M. Beneficial effect of carotid endarterectomy in symptomatic patients with high-grade carotid stenosis. N Engl J Med, 1991, 325(7):445-453. https://doi.org/10.1056/ NEJM199108153250701 PMID: 1852179

[28] European Carotid Surgery Trialists' Collaborative Group. MRC European Carotid Surgery Trial: interim results for symptomatic patients with severe $(70-99 \%)$ or with mild $(0-29 \%)$ carotid stenosis. Lancet, 1991, 337(8752):1235-1243. PMID: 1674060

[29] el-Barghouty N, Nicolaides A, Bahal V, Geroulakos G, Androulakis $A$. The identification of the high risk carotid plaque. Eur J Vasc Endovasc Surg, 1996, 11(4):470-478. https://doi. org/10.1016/s1078-5884(96)80184-5 PMID: 8846185

[30] Elatrozy T, Nicolaides A, Tegos T, Zarka AZ, Griffin M, Sabetai M. The effect of B-mode ultrasonic image standardisation on the echodensity of symptomatic and asymptomatic carotid bifurcation plaques. Int Angiol, 1998, 17(3):179-186. PMID: 9821032

[31] Popa RF, Strobescu C, Baroi G, Raza A, Fotea V. Complex utrasound study of the atherosclerotic plaque. Rev Med Chir Soc Med Nat lasi, 2013, 117(2):424-430. PMID: 24340526

[32] Strobescu-Ciobanu C, Popa RF, Giuşcă SE, Rusu A, Lupaşcu CD. Correlations between grayscale and histopathological proprieties of carotid atherosclerotic plaque. Rev Med Chir Soc Med Nat lasi, 2020, 124(1):79-85.

[33] Gray-Weale AC, Graham JC, Burnett JR, Byrne K, Lusby RJ. Carotid artery atheroma: comparison of preoperative B-mode ultrasound appearance with carotid endarterectomy specimen pathology. J Cardiovasc Surg (Torino), 1988, 29(6):676-681. PMID: 3062007

[34] Lovett JK, Redgrave JNE, Rothwell PM. A critical appraisal of the performance, reporting, and interpretation of studies comparing carotid plaque imaging with histology. Stroke, 2005, 36(5):1085-1091. https://doi.org/10.1161/01.STR.00001607 49.61763.95 PMID: 15774817

[35] Makris GC, Nicolaides AN, Geroulakos G. Histological analysis of the carotid plaque post-endarterectomy: a waste of time or a wasted piece of information? Eur J Vasc Endovasc Surg, 2011, 42(1):13-14. https://doi.org/10.1016/j.ejvs.2011.03.028 PMID: 21498091

[36] Rothwell PM, Eliasziw M, Gutnikov SA, Fox AJ, Taylor DW, Mayberg MR, Warlow CP, Barnett HJM, Carotid Endarterectomy Trialists' Collaboration. Analysis of pooled data from the randomised controlled trials of endarterectomy for symptomatic carotid stenosis. Lancet, 2003, 361(9352):107116. https://doi.org/10.1016/s0140-6736(03)12228-3 PMID: 12531577

[37] Rothwell PM, Eliasziw M, Gutnikov SA, Warlow CP, Barnett HJM, Carotid Endarterectomy Trialists Collaboration. Endarterectomy for symptomatic carotid stenosis in relation to clinical subgroups and timing of surgery. Lancet, 2004, 363(9413):915-924. https:// doi.org/10.1016/S0140-6736(04)15785-1 PMID: 15043958

[38] Tegos TJ, Sohail M, Sabetai MM, Robless P, Akbar N, Pare G, Stansby G, Nicolaides AN. Echomorphologic and histopathologic characteristics of unstable carotid plaques. AJNR Am J Neuroradiol, 2000, 21(10):1937-1944. PMID: 11110550

[39] Ratliff DA, Gallagher PJ, Hames TK, Humphries KN Webster JH, Chant AD. Characterization of carotid artery disease: comparison of duplex scanning with histology. Ultrasound Med Biol, 1985, 11(6):835-840. https://doi.org/10.1016/ 0301-5629(85)90077-8 PMID: 3913082

[40] European Carotid Plaque Study Group. Carotid artery plaque composition - relationship to clinical presentation and ultrasound B-mode imaging. Eur J Vasc Endovasc Surg, 1995, 10(1):23-30. https://doi.org/10.1016/s1078-5884(05)80194-7 PMID: 7633965

[41] Reiter M, Horvat R, Puchner S, Rinner W, Polterauer P, Lammer J, Minar E, Bucek RA. Plaque imaging of the internal carotid artery - correlation of B-flow imaging with histopathology. AJNR Am J Neuroradiol, 2007, 28(1):122-126. PMID: 17213437

[42] de Bray JM, Baud JM, Delanoy P, Camuzat JP, Dehans V, Descamp-Le Chevoir J, Launay JR, Luizy F, Sentou Y, Cales P. Reproducibility in ultrasonic characterization of carotid plaques. Cerebrovasc Dis, 1998, 8(5):273-277. https://doi.org/10.1159/ 000015865 PMID: 9712925

[43] Arnold JA, Modaresi KB, Thomas N, Taylor PR, Padayachee TS. Carotid plaque characterization by duplex scanning: observer error may undermine current clinical trials. Stroke, 1999, 30(1): 61-65. https://doi.org/10.1161/01.str.30.1.61 PMID: 9880389

[44] Denzel C, Balzer K, Müller KM, Fellner F, Fellner C, Lang W. Relative value of normalized sonographic in vitro analysis of arteriosclerotic plaques of internal carotid artery. Stroke, 2003, 34(8):1901-1906. https://doi.org/10.1161/01.STR.0000081982. 85010.A8 PMID: 12855830

[45] Meiliana A, Dewi NM, Wijaya A. Mesenchymal stem cell secretome: cell-free therapeutic strategy in regenerative medicine. Indones Biomed J, 2019, 11(2):113-124. https:// doi.org/10.18585/inabj.v11i2.839

[46] Golledge J, McCann M, Mangan S, Lam A, Karan M. Osteoprotegerin and osteopontin are expressed at high concentrations within symptomatic carotid atherosclerosis. Stroke, 2004, 35(7):1636-1641. https://doi.org/10.1161/01.STR.0000 129790.00318.a3 PMID: 15143295

[47] Kadoglou NPE, Gerasimidis T, Golemati S, Kapelouzou A, Karayannacos $\mathrm{PE}$, Liapis $\mathrm{CD}$. The relationship between serum levels of vascular calcification inhibitors and carotid plaque vulnerability. J Vasc Surg, 2008, 47(1):55-62. https://doi.org/ 10.1016/j.jvs.2007.09.058 PMID: 18178454

[48] Butler WT. The nature and significance of osteopontin. Connect Tissue Res, 1989, 23(2-3):123-136. https://doi.org/10.3109/ 03008208909002412 PMID: 2698313

[49] Icer MA, Gezmen-Karadag M. The multiple functions and mechanisms of osteopontin. Clin Biochem, 2018, 59:1724. https://doi.org/10.1016/j.clinbiochem.2018.07.003 PMID: 30003880

[50] Lok ZSY, Lyle AN. Osteopontin in vascular disease. Arterioscler Thromb Vasc Biol, 2019, 39(4):613-622. https://doi.org/ 10.1161/ATVBAHA.118.311577 PMID: 30727754 PMCID: PMC6436981

[51] Denhardt DT, Noda M, O'Regan AW, Pavlin D, Berman JS. Osteopontin as a means to cope with environmental insults: regulation of inflammation, tissue remodeling, and cell survival. J Clin Invest, 2001, 107(9):1055-1061. https://doi.org/10. 1172/JCI12980 PMID: 11342566 PMCID: PMC209291

[52] Myers DL, Harmon KJ, Lindner V, Liaw L. Alterations of arterial physiology in osteopontin-null mice. Arterioscler Thromb Vasc Biol, 2003, 23(6):1021-1028. https://doi.org/10.1161/01.ATV. 0000073312.34450.16 PMID: 12714436

[53] Chiba S, Okamoto H, Kon S, Kimura C, Murakami M, Inobe M, Matsui Y, Sugawara T, Shimizu T, Uede T, Kitabatake A. Development of atherosclerosis in osteopontin transgenic mice. Heart Vessels, 2002, 16(3):111-117. https://doi.org/10.1007/ s003800200005 PMID: 12027233

[54] Wolak T, Sion-Vardi N, Novack V, Greenberg G, Szendro G, Tarnovscki T, Nov O, Shelef I, Paran E, Rudich A. N-terminal rather than full-length osteopontin or its C-terminal fragment is associated with carotid-plaque inflammation in hypertensive patients. Am J Hypertens, 2013, 26(3):326-333. https://doi. org/10.1093/ajh/hps043 PMID: 23382482 
[55] Herisson F, Heymann MF, Chétiveaux M, Charrier C, Battaglia S Pilet P, Rouillon T, Krempf M, Lemarchand P, Heymann D, Gouëffic Y. Carotid and femoral atherosclerotic plaques show different morphology. Atherosclerosis, 2011, 216(2): 348-354. https://doi.org/10.1016/j.atherosclerosis.2011.02.004 PMID: 21367420

[56] Kim J, Song TJ, Yang SH, Lee OH, Nam HS, Kim YD, Kim EH, Lee HS, Nam CM, Heo JH. Plasma osteoprotegerin levels increase with the severity of cerebral artery atherosclerosis. Clin Biochem, 2013, 46(12):1036-1040. https://doi.org/10. 1016/j.clinbiochem.2013.05.048 PMID: 23726804

[57] Blázquez-Medela AM, García-Ortiz L, Gómez-Marcos MA Recio-Rodriguez JI, Sánchez-Rodríguez A, López-Novoa JM, Martínez-Salgado C. Osteoprotegerin is associated with cardiovascular risk in hypertension and/or diabetes. Eur $\mathrm{J}$ Clin Invest, 2012, 42(5):548-556. https://doi.org/10.1111/j. 1365-2362.2011.02619.x PMID: 22050177
[58] Halak S, Östling G, Edsfeldt A, Kennbäck C, Dencker M, Gonçalves I, Asciutto G. Spotty carotid plaques are associated with inflammation and the occurrence of cerebrovascular symptoms. Cerebrovasc Dis Extra, 2018, 8(1):16-25. https:// doi.org/10.1159/000485258 PMID: 29402768 PMCID: PMC 5836198

[59] Davaine JM, Quillard T, Brion R, Lapérine O, Guyomarch B, Merlini T, Chatelais M, Guilbaud F, Brennan MÁ, Charrier C, Heymann D, Gouëffic Y, Heymann MF. Osteoprotegerin, pericytes and bone-like vascular calcification are associated with carotid plaque stability. PLoS One, 2014, 9(9):e107642. https://doi.org/10.1371/journal.pone.0107642 PMID: 25259713 PMCID: PMC4178031

\section{Corresponding author}

Radu Florin Popa, Associate Professor, MD, PhD, Department of Vascular Surgery, Grigore T. Popa University of Medicine and Pharmacy, 16 University Street, 700115 laşi, Romania; Phone +40722-433 335, e-mail: radu.popa@umfiasi.ro

Received: May 25, 2020

Accepted: December 30, 2020 